\title{
THE DETECTION OF FLOW METER DRIFT BY USING STATISTICAL PROCESS CONTROL
}

\author{
M. BEN SALAMAH ${ }^{1}$, A. KAPOOR 2 , M. SAVSAR ${ }^{3}$, M. EKTESABI ${ }^{2}$, A. ABDEKHODAEE 2 \& E. SHAYAN \\ ${ }^{1}$ The Electric Power Section of the High Institute for Energy, \\ the Public Authority for Applied Education and Training (PAAET), Kuwait. \\ ${ }^{2}$ The Faculty of Engineering, Swinburne University of Technology, Melbourne, Australia. \\ ${ }^{3}$ The Industrial and Management Systems Engineering Section, \\ the College of Engineering \& Petroleum, Kuwait University, Kuwait. \\ ${ }^{4}$ www.Shayan.com.au.
}

\begin{abstract}
Flow meter drift is a serious problem, the financial losses from which can be huge. Flow meters do not usually get much attention and a drift can go unnoticed for a long time. In this research, a novel method is presented for the early detection of flow meter drift. The method is based on statistical process control (SPC). The method can be used with any type of flow meter (ultrasonic, magnetic etc.) regardless of its manufacturer. Another advantage of the presented method is that it does not require any knowledge of the mathematical models and relations that govern the process. The method is also capable of working with minimal data: only the monthly billing data are needed. Adapting the process can be done inexpensively.
\end{abstract}

Keywords: cusum, data analysis, flow meter, flow meter drift, statistical process control.

\section{INTRODUCTION}

The accuracy of measuring devices is of great importance in almost all aspects of human life: health care, environmental studies and billing. These devices, however, are subject to failure. The consequence of this failure varies depending on what the measuring device was employed for.

It is the supplier's ethical duty (towards the owners/share holders and towards the customers/ consumers) to make sure that the measuring devices for what is being supplied are as accurate as possible because if this is not the case, the supplier would be running the risk of suffering considerable financial loses.

To ensure the accuracy of their measuring devices, suppliers usually resort to calibration. Calibration involves the checking of a measuring instrument against accurate standards to determine any deviation and correction for errors [1]. This process, nevertheless, will not end the problem. For instance, when a measuring instrument is calibrated and found acceptable for the time being, this would not be a guarantee that it would not malfunction in the future. In fact, calibration itself might pose some problems. These problems include

- Some measuring instruments would require the supply process to be ceased in order for them to be calibrated. The supplier may incur financial losses because of the lost revenue that results from stopping production and the consumer/customer may also be disturbed because of this and search for alternatives.

- When calibration is done in-house, it requires the employment of a number of staff and the purchasing of certain equipment. Both require allocating a portion of the supplier's budget to them.

- When calibration is outsourced, it might involve sending the measuring instruments to a calibration laboratory, during which time, alternative measuring instruments would probably be installed in place of the ones sent to calibration. This, again, has its price.

- In all cases, calibration takes time and demands effort from the supplier's employees. The time taken and effort dispensed are at the expense of other tasks.

(C) 2011 WIT Press, www.witpress.com

ISSN: 1743-7601 (paper format), ISSN: 1743-761X (online), http://journals.witpress.com

DOI: $10.2495 /$ SDP-V6-N1-91-103 
Because of the aforementioned problems, calibration is almost never done during seasons or periods of high demand. It is usually done during low supply times, when some equipment, supply lines or processes can be spared and taken out of production. A measuring instrument, nevertheless, is susceptible to failure at any time. Consequently, a measuring instrument might be giving inaccurate readings during a high demand season and probably would only be discovered during a low demand season when it is already too late.

In this paper, a method for the detection of flow meter drift for pumping stations and pipe flow is presented. The method presented is by no means a substitute for periodical calibration recommended by flow meter manufacturers. It is, however, a fail-safe procedure for detecting when a drift takes place between times of calibration.

Flow meter designers and manufacturers present some hardware solutions for the problem of drift detection. Monitoring systems can also be made that compare the input variables of a pumping system to its output variables and detect any deviation from normal behavior. Making a continuous online flow meter monitoring system would be both complicated and expensive. Consequently, any method that is data intensive would probably not work. Only the monthly consumption bills were available for the study sites. As a result, the solution for the problem of flow meter drift detection had to work from the data in the monthly consumption bills only.

Of the many statistical methods available, statistical process control (SPC) was thought of as the most appropriate one. Also, of the many SPC methods, the tabular CUSUM was thought of as the most suitable method for detecting flow meter drift. Nevertheless, a serious obstacle had to be overcome. The consumption data took the form of a seasonal time series that widely oscillates (increasing in summer and decreasing in winter). This time series was not suitable for use in SPC in general and more specially in a cusum method. The cusum method is basically one that detects mean shift. An approach had to be thought of to transform the seasonal time series consumption data to a medium suitable for use in SPC-cusum.

The approach taken was creating a virtual mean, $f_{t}\left(\overline{x_{v}}\right)$. For every point in the time series (regardless of its location) there would exit a virtual point that represents the virtual mean corresponding to this point. It was this virtual mean that was processed by the cusum method. The method is explained in the research method section.

\section{LITERATURE REVIEW}

Bolton [2] defined drift by writing that 'An instrument is said to show drift if there is a gradual change in output over a period of time which is unrelated to any change in input'. Sydenham et al. [3] defined drift in a different way: 'Drift is the rate of change of the signal output with time'. As for the nature of drift and its predictability, Sydenham et al. stated that 'Drift is a complex effect usually poorly understood and seldom occurs at a predictable, fixed rate'. Sydenham et al. have also written that drift 'is often a key limiting factor on system discrimination and accuracy and cannot be ignored'.

Nilsson [4] has quoted Meshkati and Groot [5] that inaccurate measurement is responsible for more than $80 \%$ of the unaccounted-for loss in the gas supply industry. A similar phenomenon, unaccountedfor water loss, is known in the potable water industry as was shown by Johnson [6] and Hopkins [7]. From the researchers' own experience, the phenomenon exists in the cooling water industry also. In fact, the issue of flow meter inaccuracy is a problem wherever flow meters are used regardless of the fluid being measured. For example, a flow meter error that resulted in unaccounted-for loss in petroleum caused a political crisis in Kuwait [8-10]. All of these problems that have to do with flow meter inaccuracy are just a part of a grater problem, which is the accuracy of the measuring instruments in general.

The issue of flow meter accuracy was the subject of many research papers and patents. However, the majority of these have been about improving the sensing devices and electronic circuitry like that of Herwig et al. [11] and Keech [12]. Therefore, the immediate benefits of such works are for flow meter manufacturers not end users. 
Very few research or practical works have considered using statistics for detecting flow meter fault. These include the paper done by Nilsson [4] who presented a method for finding inaccurate gas flow meters using billing data. The method uses billing readings to find inaccurate meters by assigning an individual load index (LI), which was primarily affected by the climate rather than the customer's individual behavior. The individual (residential) LI is compared with an average LI, and meters that differed from the average LI were examined. The method excluded gas flow meters that were used in industrial establishments because the consumption is not affected by the climate.

As mentioned earlier, what affected gas consumption in a residential area was largely the weather. Hence, investigating outliers was a good method for finding faulty flow meters. Unfortunately, this method cannot be used in an industrial environment because industrial consumers vary greatly in their consumption. For instance, the quantity of seawater consumed in a month by one plant is more than that consumed by another in a year.

The seminal work on statistical process control is often attributed to Walter Shewhart and his book Economic Control of Quality of Manufactured Product, which was published in 1931 [13]. Several introductory works on SPC include books by Montgomery [14], Wheeler and Chambers [15], Norton [16], Amsden et al. [17], Abbott [18] and Wheeler [19].

Statistical process control is a method for monitoring the mean of a process and is a very popular method for doing this. Nevertheless, Han and Tsung [20] ascertain that the current methods of SPC focus mostly on monitoring and detection of constant shifts in the mean. Other conditions where the mean shift is dynamic have not been thoroughly studied, according to them. To fill this gap, Han and Tsung [20] designed a reference-free Cuscore (RFCuscore) chart for tracing and detecting dynamic mean changes.

The seminal work on Cumulative Sum (CUSUM) was done by Page [21]. In his paper Page explained that in the industry, with its continuous production process, the quality of the output is approximately constant. It only worsens as a result of a fault at some point in the process. Page wrote that 'In general, it will be possible to assign a quality number, $\theta$, to the output which may be taken as a parameter of the distribution. We are interested in the changes in $\theta^{\prime}$. Detecting changes in the parameter $\theta$ was done by, what was called, process inspection schemes. These schemes detected deterioration in the quality of the output from a continuous production process. A widely used scheme consists of examining samples of a fixed size at regular intervals of time. In that paper rules were developed that 'use all the observations since action was last taken and that are suitable for the detection of any magnitude of the change in the parameter' [21]. Unlike the Shewhart chart, 'With this rule the decision whether or not to take action is made after each sample and all the previous samples are used in making the decision' [21]. Page was also interested in estimating the point at which the change took place.

Later Page [22] presented a more thorough explanation of the cumulative sum charts and methods. Advancements since the publication of the first paper in 1954 up to 1961 were also presented. In 1962, Page [23] presented a research paper about using gauging as an input for the CUSUM rather than measured observations. The scheme was for controlling the mean and standard deviation of a normal distribution. Using gauging was better than the Shewhart chart for detecting small changes but slightly less than the CUSUM schemes based on observations.

The cumulative sum is made in two ways: tabular and graphic. The graphic method utilizes a V-mask. Some works on the construction of the V-mask include that of Johnson [24]. That work was about how the theory of sequential probability ratio tests can help in constructing the V-mask.

Ewan [25] outlined the various types of continuous graphical control schemes that were available (at that time) and the type of process for which cusum charts are most appropriate.

Ewan has noticed that 'the cu-sum chart is effectively a running mean chart and that it is more effective in detecting sustained changes ... than the standard control chart. On the other hand, the control chart is more effective in detecting larger, shorter term changes and is extremely simple to use' [25]. 
Just like many monitoring schemes, the purpose of the cusum method is to stop production when an alarm is given. This alarm could either be true or false. The economic cost of stopping production in both cases and the cost of running the cusum method itself was first considered by Taylor [26]. In his paper an approximate formula for the long run average cost per unit time as a function of the parameters of the cumulative sum chart was developed. The purpose of doing this was to enable these parameters to be chosen optimally under the average cost criterion. Chiu [27] had some criticisms on Taylor's method. Chiu presented a study of the economic design of cusum charts, which, according to Chiu, overcame the aforementioned criticisms. Chiu also provided a simple scheme that determines a control plan close to optimum.

More recent works about the cusum method can be found in Zhao et al. [28] who produced a dual cusum (DCUSUM) by combining two cusum charts to detect a range of mean shifts. Other examples of recent work include those by Han et al. Han et al. [29] stated that although the cusum method is simple, its performance deteriorates when the actual mean shift is unknown. To overcome this condition, an alternative approach called the CUSUM chart with local signal amplification (LSA-CUSUM) was presented. This scheme worked by amplifying or weakening local signals to improve the power of the traditional CUSUM chart in detecting an unknown mean shift over a range. Han et al. claimed that measurable weakening and amplifications of local signals can improve the ability of the CUSUM chart in detecting the local mean shift. Han et al. [30] also created a multi-chart consisting of several CUSUM or EWMA charts with different reference values that were used simultaneously to detect anticipated process changes. Their work showed that the multi-chart had the merits of quick detection of a range of mean shifts, was easy and had a flexible design for various situations and great reduction in computational complexity. Han and Tsung [31] also made a cusum multi-chart scheme consisting of multiple cusum control charts for detecting and diagnosing unknown abrupt changes in a process. They showed that this scheme performed better than the single cusum or EWMA, cusum multi-chart, EWMA multi-chart and GLR (Generalized Likelihood Ratio) charts.

\section{THE RESEARCH METHOD}

\subsection{Transforming the seasonal time series to a linear time series}

As mentioned earlier, seasonal time series are not the best medium for SPC methods in general and the tabular CUSUM specially. All SPC methods require that the process data be closely gathered around a mean. This is not the case of a seasonal time series where data are usually very far from the mean.

One of the research problems, therefore, became of finding a suitable method for transforming the seasonal time series to a linear time series. This problem is depicted in Fig. 1.

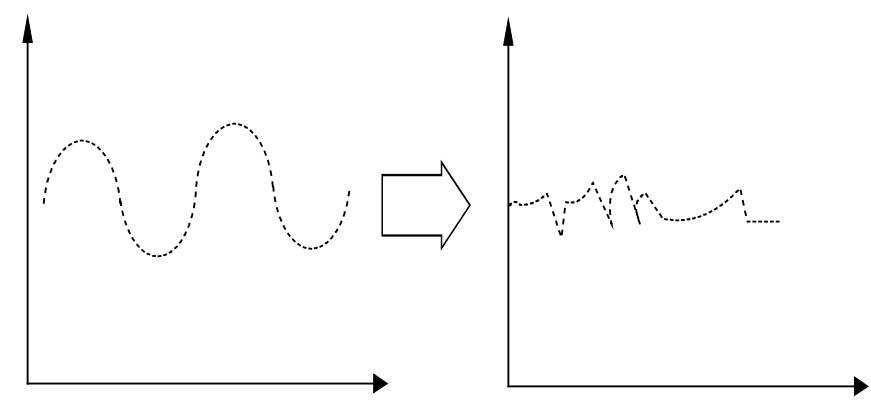

Figure 1: Transforming the seasonal time series to a linear time series. 
The transformation was achieved by exploiting two features of a sinusoidal wave and their corresponding features of seasonal time series. The explanation follows.

Let us examine a sinusoidal wave given by the function

$$
Y(\theta)=\mu+a \sin (\theta)
$$

Where $a>0$ and $\mu>0$; let us also assume that this function is sampled at equal time periods.

In every wave length (before the wave repeats itself) we assume that it has been sampled ' $d$ ' times i.e. ' $d$ ' is the number of samples in every $2 \pi$ (or $360^{\circ}$ ). Let ' $\alpha$ ' be defined as $\alpha=2 \pi n$, were $n=1,2,3$ and so on. Consequently, when the wave repeats itself again and again as $\theta$ increases, every two points that are $\mathrm{d}$ samples apart would be equal because

$$
\sin (\theta)=\sin (\theta+\alpha)
$$

This would mean that

$$
Y(\theta)=Y(\theta+\alpha)
$$

Manipulating eqn (3) makes it

$$
\mathrm{Y}(\theta)-\mathrm{Y}(\theta+a)=0
$$

A discrete seasonal time series (with no trend) looks almost like the sinusoidal function discussed earlier. It oscillates around a mean (or more precisely an estimate of the mean, $\bar{X}$ ) and repeats itself once every year. Therefore, if a seasonal time series is given by

$$
X(t)=X_{1}, X_{2}, \ldots, X_{n}
$$

and if this series is sampled d times ( $d$ could be 12 month or 4 quarters ..., etc.), then eqn (4) becomes

Or similarly

$$
X(t)-X(t+d)=0+\varepsilon_{1}
$$

$$
X(t)-X(t-d)=0+\varepsilon_{1}
$$

where $\varepsilon_{1}$ is i.i.d. (independent and identically distributed).

Another property of sinusoidal waves is that when every two points that are $\alpha / 2$ (or $\pi$ ) apart are averaged, the resulting average is the mean

$$
\frac{Y(\theta)+Y\left(\theta+\frac{a}{2}\right)}{2}=\mu
$$

Or similarly

$$
\frac{Y(\theta)+Y\left(\theta-\frac{a}{2}\right)}{2}=\mu
$$

In a seasonal time series, every two points that are $d / 2$ time periods apart are averaged; the resulting average is the estimate of the mean. Thus, eqn (8) becomes

$$
\frac{X_{t}+X_{\left(t+\frac{d}{2}\right)}}{2}=\bar{X}+\varepsilon_{2}
$$


Or similarly

$$
\frac{X_{t}+X_{\left(t-\frac{d}{2}\right)}}{2}=\bar{X}+\varepsilon_{2}
$$

where $\bar{X}$ is the estimate of the mean and $\varepsilon_{2}$ is i.i.d.

In the case of a seasonal time series, adding eqns (7) and (11) would result in the virtual or running mean for every point. We called this function $f_{t}\left(X_{v}\right)$ and it represents the virtual mean for every point.

$$
f_{t}\left(\overline{X_{v}}\right)=X_{t}-X_{(t-d)}+\frac{X_{t}+X_{\left(t-\frac{d}{2}\right)}}{2}
$$

It can be shown that the running or virtual mean given in eqn (12) equals the seasonal time series' estimated mean plus some residual.

where

$$
f_{t}\left(\overline{X_{v}}\right)=\bar{X}+\varepsilon
$$

$$
\varepsilon=\varepsilon_{1}+\varepsilon_{2}
$$

And $\varepsilon$ is i.i.d.

The form given is eqn (12) is exactly what is needed for a process to be monitored by SPC methods and, more specifically, the tabular CUSUM.

\subsection{The method of the CUSUM}

According to Montgomery [14], the cumulative sum is done by plotting the cumulative sums of the deviations of the sample values from a target value (which is usually the mean).

$$
C_{t}=\sum_{j=1}^{t}\left(x_{j}-\bar{x}\right)
$$

Or, put in another way,

$$
C_{t}=\left(x_{t}-\bar{x}\right)+C_{t-1}
$$

where $C_{t}$ is the cumulative sum up to and including the $t$ th sample; the starting value $C_{0}=0$.

There are two ways to represent cusums:

1. Tabular (or algorithmic) cusum.

2. V-mask form of the cusum.

Only the first method is going to be used.

\subsubsection{The method of tabular CUSUM}

According to Montgomery [14], the tabular cusum works by accumulating deviations from the target that are above it with one statistic $C^{+}$and accumulating deviations from the target that are below it with another statistic $C^{-}$.

If the mean is the target, then

$$
C_{t}^{+}=\max \left[0, x_{t}-(\bar{x}+k)+C_{t-1}^{+}\right]
$$




$$
C_{t}^{-}=\max \left[0,(\bar{x}-k)-x_{t}+C_{t-1}^{-}\right]
$$

Where $C_{0}{ }^{+}=C_{0}^{-}=0$, and $k$ is a reference value; $k$ is one-half the magnitude of the shift we are interested in detecting.

When the virtual mean is used, eqn (17) becomes

$$
C_{t}^{+}=\max \left[0, f_{t}\left(\overline{x_{v}}\right)-(\bar{x}+k)+C_{t-1}^{+}\right]
$$

And eqn (18) becomes

$$
C_{t}^{-}=\max \left[0,(\bar{x}-k)-f_{t}\left(\overline{x_{v}}\right)+C_{t-1}^{-}\right]
$$

In this paper the magnitude of the shift we are interested in detecting is one standard deviation $\sigma$ (of $f_{t}\left(\overline{X_{v}}\right)$ and not the original time series $X(t)$ ). Accordingly,

$$
k=\frac{\sigma}{2}
$$

Note that $C_{t}^{+}$and $C_{t}^{-}$accumulate deviations from the target value (in our case $\bar{x}$ ) that are grater than $\mathrm{k}$ with both quantities being rest to zero on becoming negative. If either $C_{t}^{+}$or $C_{t}^{-}$exceeds a decision interval, $H$, the process is considered to be out of control. Montgomery [14] suggests that a reasonable value for $H$ is five times the process standard deviation.

$$
H=5 \sigma
$$

In this research paper we followed Montgomery's suggestion.

\section{CASE STUDIES AND RESULTS}

In this section, two case studies are given.

\subsection{Case study \# 1}

This was a documented case of flow meter drift. The flow meter readings of a plant having the code name C7 is given in Table 1 and is shown in Fig. 2.

It can be seen that the flow meter readings for the consumption were decreasing over the years. At that time it was thought that the decrease in consumption was due to C7's economy in the use of seawater and not to a flow meter fault. What led to this belief was that the owners of C7 expressed that they were taking measures to decrease the use of cooling seawater. Simultaneously, a decline in the flow meter readings was being noticed.

Suspicions in the abnormality of the plant consumption only started when it was, coincidently, noticed that the pressure produced by operating pumps (for C7) should give more flow than what was recorded by the flow meter. This meant one of three possibilities:

1. the pressure gauges were wrong, or

2. the flow meter was wrong, or

3. there was a major leak in the pipeline.

The pressure gauges were calibrated and were found to be in a good condition and there was no leak in the pipeline. This made the flow meter the only suspect. When the flow meter in question was inspected and calibrated, it became clear that it was inaccurate. This meant that C7 did not use less water during that period and its efforts to save water were a failure. The proof of this is the 'jump' in 
Table 1: Recorded readings for C7.

\begin{tabular}{lrrrrrrr}
\hline Month & Consumption & \multicolumn{1}{c}{ Month } & Consumption & Month & Consumption & Month & Consumption \\
\hline Mar-99 & $12,046,300$ & Jan-01 & $12,233,800$ & Nov-02 & $11,711,000$ & Sep-04 & $8,969,000$ \\
Apr-99 & $12,151,500$ & Feb-01 & $11,519,300$ & Dec-02 & $11,091,500$ & Oct-04 & $9,434,700$ \\
May-99 & $12,783,100$ & Mar-01 & $13,684,700$ & Jan-03 & $10,430,300$ & Nov-04 & $8,235,300$ \\
Jun-99 & $12,506,400$ & Apr-01 & $13,133,600$ & Feb-03 & $8,813,200$ & Dec-04 & $7,366,200$ \\
Jul-99 & $13,446,300$ & May-01 & $13,816,100$ & Mar-03 & $9,436,600$ & Jan-05 & $6,730,900$ \\
Aug-99 & $14,095,300$ & Jun-01 & $13,490,000$ & Apr-03 & $10,867,500$ & Feb-05 & $6,151,100$ \\
Sep-99 & $12,695,200$ & Jul-01 & $13,538,100$ & May-03 & $11,562,800$ & Mar-05 & $5,924,900$ \\
Oct-99 & $13,348,600$ & Aug-01 & $13,324,800$ & Jun-03 & $11,219,100$ & Apr-05 & $5,912,600$ \\
Nov-99 & $12,361,700$ & Sep-01 & $12,537,700$ & Jul-03 & $10,707,800$ & May-05 & $11,402,400$ \\
Dec-99 & $10,362,000$ & Oct-01 & $14,092,700$ & Aug-03 & $9,648,700$ & & \\
Jan-00 & $11,768,700$ & Nov-01 & $13,293,200$ & Sep-03 & $9,648,700$ & & \\
Feb-00 & $10,615,000$ & Dec-01 & $9,631,400$ & Oct-03 & $9,669,500$ & & \\
Mar-00 & $12,069,800$ & Jan-02 & $13,243,200$ & Nov-03 & $8,901,200$ & & \\
Apr-00 & $12,238,400$ & Feb-02 & $11,900,000$ & Dec-03 & $8,552,600$ & & \\
May-00 & $12,847,700$ & Mar-02 & $11,753,096$ & Jan-04 & $8,142,600$ & & \\
Jun-00 & $13,376,000$ & Apr-02 & $10,546,200$ & Feb-04 & $8,026,100$ & & \\
Jul-00 & $13,897,700$ & May-02 & $12,292,500$ & Mar-04 & $8,728,800$ & & \\
Aug-00 & $15,004,100$ & Jun-02 & $11,910,500$ & Apr-04 & $8,448,400$ & & \\
Sep-00 & $14,279,400$ & Jul-02 & $12,135,500$ & May-04 & $9,717,900$ & & \\
Oct-00 & $13,940,400$ & Aug-02 & $12,408,800$ & Jun-04 & $9,949,700$ & & \\
Nov-00 & $12,888,000$ & Sep-02 & $12,442,100$ & Jul-04 & $10,647,200$ & & \\
Dec-00 & $12,825,600$ & Oct-02 & $12,446,000$ & Aug-04 & $12,583,400$ & & \\
\hline
\end{tabular}

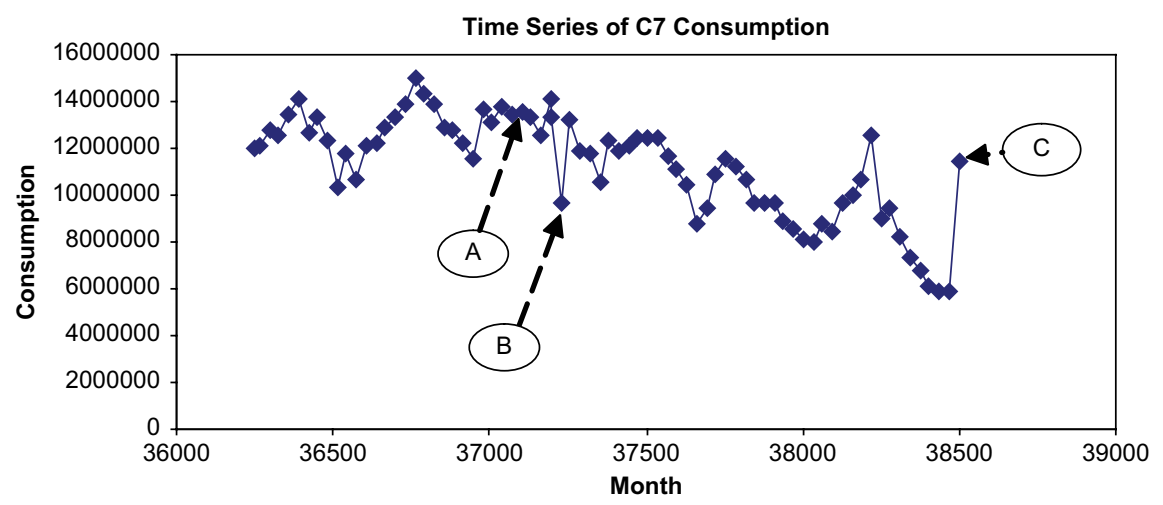

Figure 2: The flow meter readings of $\mathrm{C} 7$.

flow meter readings after calibration (the flow meter drift was discovered in the first week of May 2005, the point C of Fig. 2).

The authors of this paper wanted to investigate the application of the tabular CUSUM method on this case to see if it would detect the drift and to determine the time of the drift's onset. The statistical properties of the virtual mean and the parameters of the tabular CUSUM for this specific consumer are shown in Table 2. 
Table 2: The statistical properties of the virtual mean and the parameters of the tabular CUSUM for C7.

\begin{tabular}{lr}
\hline Mean (X bar) & $13,566,054$ \\
Standard Deviation & 915,876 \\
K & 457,938 \\
Xbar+K & $14,023,992$ \\
Xbar-K & $13,108,116$ \\
H & $4,579,380$ \\
\hline
\end{tabular}

The tabular CUSUM method was able to detect the drift on December 2001 (point B in Fig. 2). The method also indicates that the start of the drift happened some time in July 2001 (point A of Fig. 2). What is interesting about this particular case is that the records show a calibration took place in October 2004. It may be concluded that this calibration was not perfect. If the method was applied, it would have detected the drift in December 2001 and saved the organization from losing an estimated $\$ 2,330,000$.

\subsection{Case study \#2}

This case study is about a plant with the code name C12. Its recorded flow meter readings for consumption are shown in Table 3. The statistical properties of the virtual mean and the parameters of the tabular CUSUM for this specific consumer are shown in Table 4. Because this consumer had a partial shutdown in November and December 2006, the consumptions of the same months of 2005 were used in the analysis. The application of the CUSUM method to C12 showed the existence of a drift that started in December 2007 (point A in Fig. 3) and was detectable in May 2008 (point B in Fig. 3) but it was not verified if it was due to flow meter error or change in the consumption behavior. However, if the drift was true, the estimated loss up to November 2008 would be $\$ 600,000$.

\section{DISCUSSION}

In analyzing many cases including the previous two, an 'OK' status was exhibited after many 'Alarm' statuses. The reason for this was that the virtual mean was calculated by using data of the same month from the previous year and of a month preceding it by six months. Because of this, when a drift is detected and an alarm status is declared but nothing is done, the virtual mean itself becomes 'corrupted'. This, in turn, would result in 'OK' status(es) after many 'Alarm' statuses. The drift detection method was applied on data that were generated before its conception. Hence, the pseudo inconsistency of producing 'OK' statuses after many 'Alarm' statuses has occurred.

As mentioned previously, the method presented has many advantages. However, this method has limitations and drawbacks. The first limitation is that (in time series terms) it needs at least two d's of data (in our case two years) before it starts functioning. Obviously, this would not make it applicable to new plants. The second limitation is that it works with seasonal time series only. Other consumption patterns such as linear and mixed seasonal and linear time series cannot have their drift detected by this method. The potable (municipal) water industry, for example, has a consumption pattern that is usually mixed linear and seasonal. Finally, the time between the onset of drift and its detection is in the order of months. In many situations, this may not be acceptable.

Expanding the limitations and eliminating the drawbacks of the presented method might be the subject of further studies. A method might be introduced that does not involve the long waiting period before application of the method previously presented. Other improvements might be done 


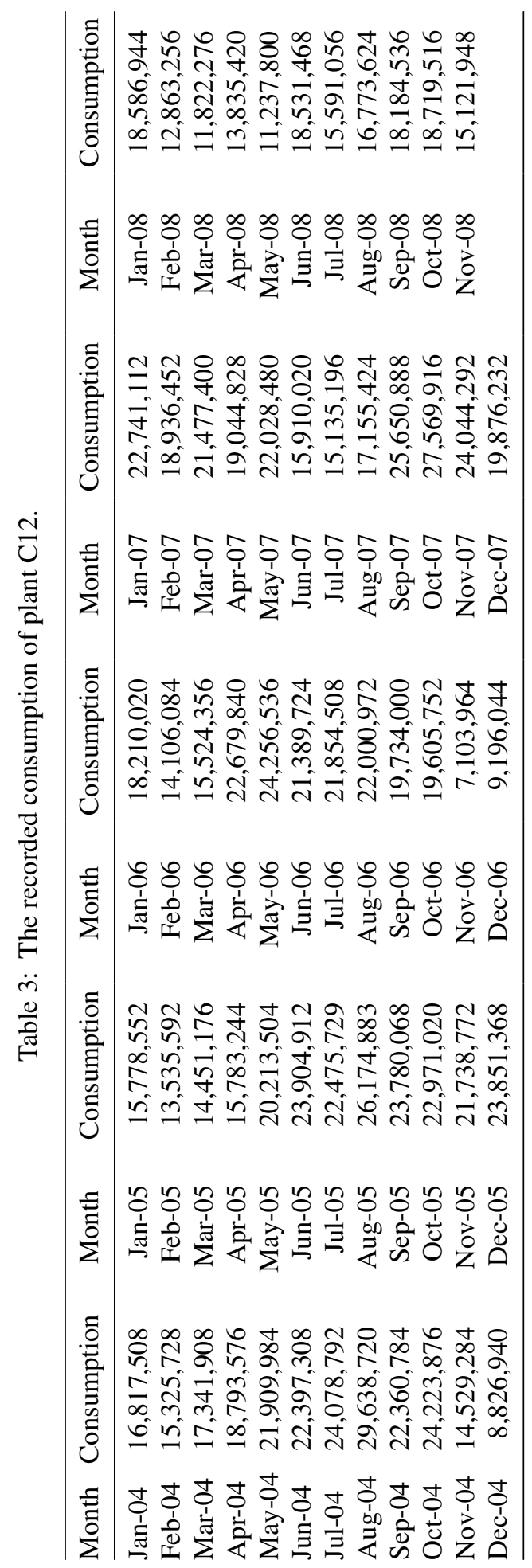


Table 4: The statistical properties of the virtual mean and the parameters of the tabular CUSUM for C12.

\begin{tabular}{lr}
\hline Mean (X bar) & $20,367,209$ \\
Standard deviation & $6,737,318$ \\
K & $3,368,659$ \\
X bar+K & $23,735,868$ \\
X bar-K & $16,998,550$ \\
H & $33,686,588$ \\
\hline
\end{tabular}

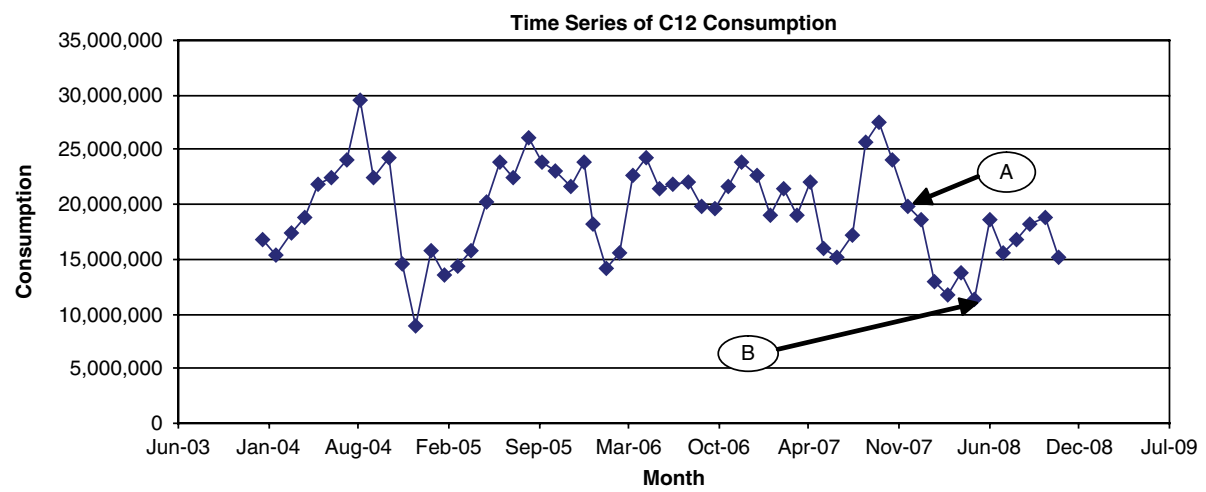

Figure 3: The flow meter readings of $\mathrm{C} 12$.

on making the method applicable on other time series such as linear and mixed linear and seasonal time series. Certainly, the issue of the time between the onset of drift and its detection can be the subject of a study aiming to shorten it.

\section{CONCLUSION}

Flow meter drift is a problem that flow meter owners face every now and then. The physical problem will have financial consequences if it happened to flow meters that are used in billing. Flow meter drift is a part of a wider phenomenon, which is instrument drift. This phenomenon is poorly understood and can happen at any time. The purpose of this paper was to present a practical and an inexpensive method to detect flow meter drift. The method presented (SPC-CUSUM) only needed the monthly billing data. From this data only, the flow meters were investigated for drift. The nature of the data, nevertheless, was an obstacle for applying the SPC-CUSUM. Therefore, before sending these data to be processed by the SPC-CUSUM, they were transformed first to what was termed as a virtual mean. The method was tested against a case that was known as a case of flow meter drift and it succeeded both in detecting the drift and in determining the time of its onset. The limitations of the method were also mentioned.

\section{ACKNOWLEDGEMENT}

The authors would like to thank the reviewers for their useful suggestions.

\section{REFERENCES}

[1] Encarta, Calibration, Microsoft Corporation, 2004.

[2] Bolton, W., Newnes Instrumentation \& Measurement Pocket Book, 3rd edn, Newnes: Oxford, 2000. 
[3] Sydenham, P.H., Hancock, N.H. \& Thorn, R., Introduction to Measurement Science and Engineering. Wiley Series in Measurement Science and Technology, ed. P.H. Sydenham, John Wiley \& Sons: New York, 1989.

[4] Nilsson, U.R.C., A new method for finding inaccurate gas flow meters using billing data: finding faulty meters using billing data. Flow Measurement and Instrumentation, 9(4), pp. 237-242, 1998. doi:10.1016/S0955-5986(98)00026-0

[5] Meshkati, S. \& Groot, J., A Study of the 1991 Unaccounted-for Gas Volume at the Southern California Gas Company, vol. III Measurement, 1993.

[6] Johnson, P.V., Unaccounted-for water puzzle: more than just leakage. Florida Water Resources Journal, 1996.

[7] Hopkins, D., Savage, P.F. \& Foxt, E., Problems encountered during research into flow rate, pattern of water consumption and unaccounted-for water losses in urban areas. Flow Measurement and Instrumentation, 6(3), pp. 173-179, 1995. doi:10.1016/0955-5986(95)00004-6

[8] AlHermi, K.A., The Lost Barrels (in Arabic) in AlQabas, Kuwait, 2007.

[9] AlJasem, M.A., The Petrolum Flow meter is Faulty! (in Arabic), 2007.

[10] AlJasem, M.A., Good! The Pipe is not Punctured but the Flow meter is Fualty! (in Arabic), Kuwait, 2007.

[11] Herwig, J., et al., Magnetic Flowmeter with Fault Detection, U.S.P. Office, Patent Number: $5,370,000,1994$.

[12] Keech, R., Flowmeter Fault Detection, U.S.P. Office, Patent Number: 6,843,137, 2005.

[13] ASQ, Walter A. Shewhart Father of statistical quality control.

[14] Montgomery, D.C., Introduction to Statistical Quality Control, John Wiley \& Sons Inc., USA, 2005.

[15] Wheeler, D.J. \& Chambers, D.S., Understanding Statistical Process Control, 2nd edn ed., SPC Press: Knoxville, Tennessee, 1992.

[16] Norton, M., A Quick Course In Statistical Process Control, Technology Skills, Upper Saddle River, Pearson Prentice Hall: New Jersey, 2005.

[17] Amsden, R.T., Butler, H.E. \& Amsden, D.M., SPC Simplified: Practical Steps to Quality, 2nd edn, Productivity Press: New York, 1998.

[18] Abbott, J.C., SPC Practical Understanding of Capability by Implementing Statistical Process Control, 3rd edn ed., Walkabout Series, Robert Houston Smith Publishers: Easley, S.C., 1999.

[19] Wheeler, D.J., Advanced Topics in Statistical Process Control the Power of Shewharts Charts. 2nd edn ed., SPC Press: Knoxville, Tennessee, 2004.

[20] Han, D. \& Tsung, F., A reference-free cuscore chart of dynamic mean change detection and a unified framework for charting performance comparison. Journal of the American Statistical Association, 101(473), pp. 368-386, 2006. doi:10.1198/016214505000000556

[21] Page, E.S., Continuous inspection schemes. Biometrika, 41(1/2), pp. 100-115, 1954. doi:10.2307/2333009

[22] Page, E.S., Cumulative sum charts. Technometrics, 3(1): pp. 1-9, 1961. doi:10.2307/1266472

[23] Page, E.S., Cumulative sum schemes using gauging. Technometrics, 4(1), pp. 97-109, 1962. doi:10.2307/1266175

[24] Johnson, N.L., A simple theoretical approach to comulative sum control charts. Journal of the American Statistical Association, 56(296), pp. 835-840, 1961. doi:10.2307/2281998

[25] Ewan, W.D., When and how to use Cu-Sum charts. Technometrics, 5(1), pp. 1-22, 1963. doi:10.2307/1266488 
[26] Taylor, H.M., The economic design of cumulative sum control charts. Technometrics, 10(3), pp. 479-488, 1968. doi:10.2307/1267102

[27] Chiu, W.K., The econmic design of cusum charts for controlling normal means. Applied Statistics, 23(3), pp. 420-433, 1974. doi:10.2307/2347134

[28] Zhao, Y., Tsung, F. \& Wang, Z., Dual CUSUM control schemes for detecting a range of mean shifts. IIE Transactions, 37(11), pp. 1047-1057, 2005. doi:10.1080/07408170500232321

[29] Han, D., Tsung, F. \& Li, Y., A cusum chart with local signal amplification for detecting a range of unknown shifts. International Journal of Reliability, Quality and Safety Engineering, 14(2), pp. 81-97, 2007. doi:10.1142/S0218539307002520

[30] Han, D., et al., Cusum and EWMA multi-charts for detecting a range of mean shifts. Statistica Sinica, 17(3), pp. 1139-1164, 2007.

[31] Han, D. \& Tsung, F., Detection and diagnosis of unknown abrupt changes using CUSUM multi-chart schemes. Sequential Analysis, 26(3), pp. 225-249, 2007. doi:10.1080/07474940701404765 